\title{
Eficacia clínica de azatioprina (AZA) en el tratamiento de colitis ulcerativa (CU) leve a moderada, con respuesta inadecuada a manejo con esteroides
}

\author{
Clinical Efficacy of Azathioprine (AZA) for Treatment of Mild to Moderate \\ Ulcerative Colitis (UC) in Patients Who Have Responded Inadequately to Steroids
}

Fabián Juliao B, MD, ${ }^{1}$ Yineth Agudelo Z, MD, ${ }^{2}$ Carlos Yepes D, MD, ${ }^{3}$ Luis I. Bejarano, MD, ${ }^{4}$ Jose G. Thorrens, MD, ${ }^{5}$ Fabián Jaimes B, MD. ${ }^{6}$

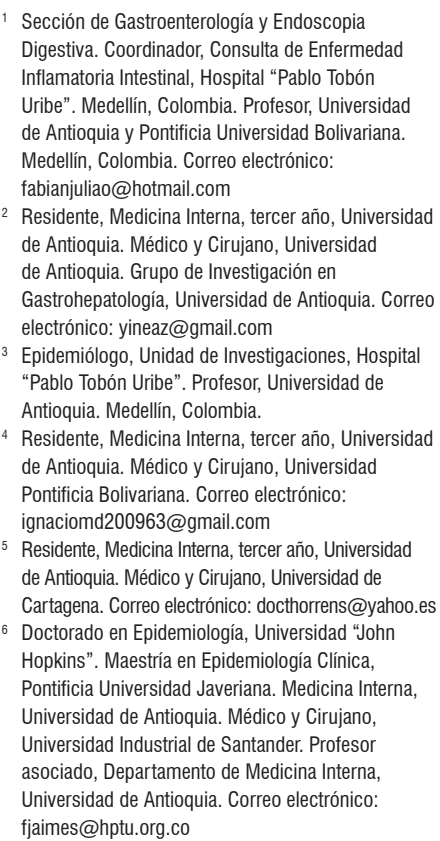

Sección de Gastroenterología y Endoscopia Digestiva. Coordinador, Consulta de Enfermedad Inflamatoria Intestinal, Hospital "Pablo Tobón Uribe". Medellín, Colombia. Profesor, Universidad de Antioquia y Pontificia Universidad Bolivariana. Medellín, Colombia. Correo electrónico: fabianjuliao@hotmail.com

Residente, Medicina Interna, tercer año, Universidad de Antioquia. Médico y Cirujano, Universidad de Antioquia. Grupo de Investigación en Gastrohepatología, Universidad de Antioquia. Correo electrónico: yineaz@gmail.com

3 Epidemiólogo, Unidad de Investigaciones, Hospital "Pablo Tobón Uribe". Profesor, Universidad de Antioquia. Medellín, Colombia.

${ }_{4}$ Residente, Medicina Interna, tercer año, Universidad de Antioquia. Médico y Cirujano, Universidad Pontificia Bolivariana. Correo electrónico: ignaciomd200963@gmail.com

5 Residente, Medicina Interna, tercer año, Universidad de Antioquia. Médico y Cirujano, Universidad de Cartagena. Correo electrónico: docthorrens@yahoo.es

${ }^{6}$ Doctorado en Epidemiología, Universidad "John Hopkins". Maestría en Epidemiología Clínica, Pontificia Universidad Javeriana. Medicina Interna, Universidad de Antioquia. Médico y Cirujano, Universidad Industrial de Santander. Profesor asociado, Departamento de Medicina Interna, Universidad de Antioquia. Correo electrónico: fjaimes@hptu.org.co

Fecha recibido: $\quad 09-02-15$ Fecha aceptado: 21-07-15

\begin{abstract}
Resumen
Introducción: los estudios de azatioprina (AZA) en el tratamiento de colitis ulcerativa (CU) son escasos y la mayoría tiene más de una década. Es necesario establecer la eficacia de AZA en nuestro medio, en sujetos con respuesta inadecuada a esteroides. Objetivo: evaluar en nuestra población la respuesta clínica a AZA, tanto a corto como a largo plazo, en CU con respuesta inadecuada a esteroides. Materiales y métodos: se realizó un estudio descriptivo retrospectivo, revisando historias clínicas entre agosto de 2001 y mayo de 2014 de 215 pacientes con CU incluidos en nuestra base de datos del Hospital "Pablo Tobón Uribe", de los cuales 69 (32\%) habían recibido AZA. 30 pacientes recibieron al menos 3 meses de tratamiento con AZA en dosis óptima de al menos $2,0 \mathrm{mg} / \mathrm{kg}$, por respuesta inadecuada a esteroides, y fueron incluidos en el estudio. Resultados: el seguimiento promedio de los pacientes fue de 20 meses (3 meses-72 meses). Se encontró remisión clínica en $17(57 \%)$ de 30 pacientes y respuesta parcial en 12 de 30 sujetos (40\%) durante 3 meses de tratamiento. Al año, de los 30 pacientes iniciales, 16 (53\%) mantenían remisión clínica, $3(10 \%)$ respuesta parcial y $5(17 \%)$ presentaron recaídas y recibieron terapia biológica. 16 pacientes (53\%) lograron suspensión de esteroides al año de seguimiento. Ningún paciente requirió colectomía. Conclusiones: este estudio demuestra una tasa de remisión clínica de 53\% con AZA en CU leve a moderada al año de seguimiento, logrando suspender esteroides en el $53 \%$ de los pacientes. AZA es una opción terapéutica de bajo costo y segura, que puede considerarse en este grupo de pacientes previo al inicio de terapia biológica.
\end{abstract}

\section{Palabras clave}

Colitis ulcerativa, azatioprina, remisión clínica, suspensión de esteroides.

\begin{abstract}
Introduction: Because there are very few studies of the use of azathioprine (AZA) for treatment of ulcerative colitis, and most are more than a decade old, we need to establish the efficacy of AZA in our environment for patients who have responded inadequately to steroids. Objective: The objective of this study is to evaluate short and long term clinical responses to AZA by ulcerative colitis patients in our population who have had inadequate responses to treatment with steroids. Materials and Methods: This is a retrospective study based on a database review of the medical records of 215 ulcerative colitis patients treated at the Hospital Pablo Tobon Uribe between August 2001 and May 2014. Sixty-nine patients (32\%) had received AZA, and 30 patients had received at least 3 months of treatment with AZA at the optimal dose of at least $2.0 \mathrm{mg} / \mathrm{kg}$ after having responded inadequately to treatment with steroids. This group was included in the study. Results: The median follow-up time was 20 months with a range from three months to 72 months. After three months of treatment, clinical remission was found in 17 patients $(57 \%)$ out of 30 patients, and partial responses were found in 12 out of 30 subjects (40\%). A year after the initial follow-up, 16 patients (53\%) maintained clinical remission, three (10\%) continued to have partial responses, and five $(17 \%)$ had relapsed and had received biological therapy. Sixteen patients (53\%) achieved one year with steroid treatment suspended and no patients required colectomies. Conclusions: This study demonstrates a clinical remission rate of $53 \%$ for patients with mild to moderate ulcerative colitis who were treated with AZA. One year follow-ups showed that continuous suspension of steroid treatment had been achieved in $53 \%$ of patients. AZA is an inexpensive and safe therapeutic option which can be considered prior to initiating biological therapy for these patients.
\end{abstract}

\section{Keywords}

Ulcerative colitis, azathioprine, clinical remission, steroid suspension. 


\section{INTRODUCCIÓN}

La colitis ulcerativa $(\mathrm{CU})$ es una patología inflamatoria crónica cuya etiología es multifactorial, caracterizada por erosiones y/o ulceración de la mucosa colónica, sangrado rectal y diarrea. Una publicación nuestra (1), en 202 pacientes con diagnóstico de enfermedad inflamatoria intestinal (EII), encontró una proporción mayor de pacientes con CU (80,7\%), comparados con enfermedad de Crohn $(15,8 \%)$ (relación 4,9:1), con una distribución similar por género, lo cual es un poco diferente a lo observado en países industrializados donde la proporción de las 2 entidades es muy similar. El manejo farmacológico de pacientes con $\mathrm{CU}$ se realiza en forma escalonada con mesalazina (5-ASA), esteroides e inmunosupresores tipo tiopurinas como la azatioprina, y últimamente con inhibidores del factor de necrosis tumoral $\alpha$ (anti-FNT) como infliximab y adalimumab (2-4). Sin embargo, la dependencia de esteroides es un problema clínico importante ( $22 \%$ a 1 año) (5), y la probabilidad de colectomía a 5 años en pacientes con ausencia de respuesta a este manejo convencional es alta, siendo 9\% para el paciente con proctitis y $35 \%$ cuando el compromiso es extenso (6).

La azatioprina (AZA) es una tiopurina que interfiere en el metabolismo de los ácidos nucleicos, donde actúa como un antagonista competitivo que produce inmunosupresión por medio de una reducción de la proliferación celular, entre otros mecanismos (7). A diferencia de la CU, el tratamiento con AZA o 6-mercaptoruina (6-MP) en enfermedad de Crohn (EC) está bien apoyado por evidencia clínica disponible en la literatura universal. En CU hay pocos datos sobre eficacia, los estudios son escasos, la mayoría de hace más de una década y de baja calidad (8-10). No existen estudios en Latinoamérica que demuestren su utilidad en el manejo de $\mathrm{CU}$, siendo un medicamento relativamente de bajo costo en nuestro medio comparado con la terapia biológica con infliximab o adalimumab. Al tener en cuenta todo lo anterior y con base en la experiencia que se tiene en nuestro centro en el manejo de la EII, se diseñó un estudio de cohorte retrospectivo y descriptivo con el objetivo principal de describir la eficacia tanto a corto como a largo plazo de AZA en pacientes con CU con respuesta inadecuada a esteroides.

\section{MATERIALES Y MÉTODOS}

\section{Tipo de estudio}

Se realizó un estudio descriptivo retrospectivo en el Hospital "Pablo Tobón Uribe", revisando historias clínicas de pacientes con CU entre agosto de 2001 y julio de 2013.

\section{Área de estudio}

El Hospital "Pablo Tobón Uribe" se encuentra en la ciudad de Medellín, es un hospital de alta complejidad, cubre un gran porcentaje de la población de la ciudad y es centro de referencia de patologías de difícil manejo como la EII. Desde el año 2001, el hospital creó la Clínica de EII debido a un interés especial de los autores de este estudio en esta patología y con el objetivo de atender en dicho espacio solo pacientes con CU y EC, con el fin de convertirnos en centro de referencia en la ciudad para el manejo de estas entidades.

\section{Población de estudio}

Se revisaron las historias clínicas de todos los pacientes con CU mayores de 18 años que recibieron AZA por lo menos 3 meses, en dosis terapéutica de al menos $2 \mathrm{mg} / \mathrm{kg}$ de peso. La presentación de AZA en nuestro país es tabletas de $50 \mathrm{mg}$ y la dosis total se formula en una dosis única diaria. El diagnóstico de CU se determinó de acuerdo con los criterios adoptados por la Organización Europea de Enfermedad de Crohn y Colitis Ulcerativa (ECCO) (11) y los pacientes se clasificaron de acuerdo con la Clasificación de Montreal (12). La indicación de inicio de AZA: pacientes con CU dependientes o refractarios a esteroides.

Los criterios diagnósticos definitivos de la $\mathrm{CU}$ se basan en la presencia de 3 de los siguientes 4 criterios, después de la exclusión de patología infecciosa, isquémica y neoplásica (11): 1 . Historia típica con diarrea y/o sangrado y/o moco en las deposiciones por más de 6 semanas o en episodios repetidos; 2. Hallazgos típicos en colonoscopia con mucosa granular, friable, con o sin ulceraciones; 3 . Hallazgos histológicos compatibles con EII dado por inflamación aguda o crónica, con criptitis y distorsión de criptas, asociado con infiltrado linfoplasmocitario, sin granulomas; 4. No sospecha de EC por estudios radiológicos de intestino delgado, ileocolonoscopia o biopsias. La severidad endoscópica y la extensión de la CU se determinaron por la colonoscopia más reciente al inicio de la azatioprina, y la extensión se definió según la clasificación de Montreal.

\section{Criterios de inclusión}

Pacientes mayores de 18 años con diagnóstico de CU que recibieran por lo menos 3 meses de tratamiento con AZA en dosis de al menos $2 \mathrm{mg} / \mathrm{kg}$ de peso, y que la indicación fuera una respuesta inadecuada a esteroides, bien sea dependencia o refractariedad al manejo con estos medicamentos, definido como (13):

- Pacientes que no han podido reducir con éxito los esteroides por debajo del equivalente a $10 \mathrm{mg}$ de predni- 
sona por día dentro de los 3 meses (12 semanas) del inicio de los corticoides.

- Pacientes que presentan una recaída en menos de 3 meses luego de la suspensión del esteroide.

- Pacientes que no han respondido al tratamiento con esteroides orales $(0,75$ a $1 \mathrm{mg} / \mathrm{kg} /$ día de prednisona oral o su equivalente) por más de 4 semanas.

\section{Criterios de exclusión}

- Pacientes en los que por diferentes circunstancias no se pudiera obtener información de su historia clínica.

- Uso previo o concomitante de terapia biológica con infliximab o adalimumab, con AZA.

- Pacientes con colectomía previa al inicio de AZA.

- Pacientes con CU aguda severa.

\section{Medición de desenlaces y definiciones}

Desenlace primario: proporción de pacientes dependientes de esteroides con respuesta sostenida libre de esteroides a AZA a 12 meses después del inicio de la misma. La respuesta sostenida se definió como el mantenimiento de la remisión clínica (puntaje de Mayo modificado <2) con suspensión completa de esteroides, y un mínimo de 6 meses sin la necesidad de volver a introducir los esteroides.

Desenlaces secundarios:

- No respuesta clínica: no disminución del puntaje de Mayo en 3 puntos o del 30\%, o incremento en el puntaje de Mayo.

- Respuesta parcial: pacientes que logran disminución del puntaje de Mayo en 3 puntos o del $30 \%$, pero no alcanzan puntaje de Mayo modificado $<2$.

- Efectos adversos de AZA: aparición dentro de las 4 semanas posteriores al inicio del tratamiento con AZA, de cualquiera de las siguientes: recuento de plaquetas $<100000$, leucocitos $<3000$, elevación de las transaminasas o bilirrubinas más de 2 veces con respecto al valor de referencia, aparición de pancreatitis aguda, infecciones oportunistas o linfoma en cualquier momento durante el seguimiento.

Se utilizó el marcador de la clínica Mayo (14), que incluye 4 tópicos: número de deposiciones, presencia de sangrado, valoración global por el médico, y la severidad endoscópica. Se define como remisión a un puntaje $\leq 2$, respuesta parcial a una disminución del $30 \%$ o de 3 puntos del puntaje total, y cicatrización mucosa a un puntaje endoscópico $<$ o igual a 1. Para la respuesta clínica a 1 año de seguimiento, se utilizó el marcador de la clínica Mayo modificado sin incluir el puntaje endoscópico. La falla al tratamiento con AZA se define como la suspensión del tratamiento por intolerancia, pérdida de respuesta clínica o cambio a terapia biológica. Se define como respuesta clínica sostenida los pacientes que no han presentado falla a AZA durante el seguimiento.

Se diseñó una base de datos en Excel, en la cual se recolectó la siguiente información de cada paciente para el análisis: 1. Edad al diagnóstico de CU; 2. Edad actual; 3. Género del paciente; 4. Localización y severidad de la colitis ulcerativa (clasificación de Montreal); 5. Puntaje de Mayo al inicio del tratamiento con azatioprina; 6. Dosis de esteroides al inicio de AZA; 7. Dosis de inicio de AZA; 8. Efectos adversos asociados con la azatioprina (trombocitopenia, leucopenia, alteración de función hepática, pancreatitis aguda, linfoma, infecciones oportunistas); 9. Proporción de pacientes con suspensión de esteroides; 10. Tasa de colectomía; 11. Uso de terapia biológica; y 12. Porcentaje de recaídas durante el seguimiento.

\section{Análisis estadístico}

Se realizó un análisis estadístico descriptivo, las variables cualitativas se expresaron por medio de frecuencias y proporciones, y las cuantitativas se describieron mediante su respectiva media y desviación estándar (DE), o la mediana (Me) y rango intercuartil (RIC) según su distribución, la cual fue verificada con la prueba de Shapiro-Wilk. Con respecto al análisis bivariado, se compararon entre sí variables cualitativas con la prueba Chi2 de Pearson o la prueba exacta de Fisher.

\section{Aspectos éticos}

El protocolo de este estudio se sometió con anterioridad al juicio del Comité de investigaciones y ética en investigaciones del Hospital "Pablo Tobón Uribe", donde fue aprobado. Se garantizó la confidencialidad de la información y no fue necesaria la realización de consentimiento informado por no implicar intervención adicional en los pacientes y recoger la información de registros clínicos en forma retrospectiva, siendo esta una investigación "Sin Riesgo" según el artículo 11 de la Resolución 8430 de 1993 del Ministerio de Salud de Colombia.

\section{RESULTADOS}

De un total de 208 pacientes con CU registrados en nuestra base de datos, 69 recibieron AZA (33\%). 30 de los 69 pacientes cumplían con los criterios de inclusión previamente señalados y se pudo obtener información completa en su historia clínica. Los 30 pacientes estaban recibiendo esteroides al inicio del tratamiento con AZA y todos recibían manejo concomitante con mesalazina. En cuanto 
al sexo, $47 \%$ eran hombres y $53 \%$ mujeres. La edad promedio de inicio de azatioprina fue de 43 años, el $80 \%$ de los sujetos presentaban edad entre 20 y 60 años. Según la clasificación de Montreal, el 57\% de los pacientes presentaban colitis extensa, el 33\% colitis izquierda y solo el 10\% tenía compromiso localizado en el recto. En la tabla 1 se presenta un resumen de las características demográficas de los pacientes.

Tabla 1. Características de los pacientes del estudio.

\begin{tabular}{lcc}
\hline \multicolumn{1}{c}{ Características } & Frecuencia & Porcentaje (\%) \\
\hline Sexo & & \\
\hline Femenino & 16 & 53 \\
Masculino & 14 & 47 \\
\hline Edad & & \\
\hline 20-40 años & 15 & 50 \\
41-60 años & 10 & 33 \\
$>$ >1 años & 5 & 17 \\
\hline Mediana 42 años & 43 & \\
\hline Clasificación de Montreal & & \\
\hline Extensión: & & 10 \\
Proctitis & 3 & 33 \\
Colitis izquierda & 10 & 57 \\
Colitis extensa & 17 & \\
\hline Años desde el diagnóstico al uso de AZA & 70 \\
\hline 1-3 años & 21 & 20 \\
3-6 años & 6 & 30 \\
>6 años & 3 & 30 \\
\hline Meses con uso de AZA & & \\
\hline 3-6 meses & 5 & \\
Hasta 12 meses & 6 & \\
Hasta 24 meses & 9 & \\
Hasta 72 meses & 9 & \\
\hline 72 meses & & \\
\hline
\end{tabular}

El seguimiento promedio de los pacientes fue de 20 meses ( 3 meses-72 meses). En cuanto a la actividad de la CU, se encontró remisión clínica en 17 (57\%) de 30 pacientes y respuesta parcial en 12 de 30 sujetos (40\%); a 3 meses de tratamiento, 1 paciente (3\%) no presentó respuesta primaria y requirió terapia biológica (figura 1). $\mathrm{Al}$ año, de los 30 pacientes iniciales, 16 (53\%) mantenían remisión clínica, $3(10 \%)$ respuesta parcial y $5(17 \%)$ presentaron recaída y recibieron terapia biológica (figura 2 ). 16 pacientes $(53 \%)$ lograron suspensión de esteroides al año de seguimiento. Solo 10 pacientes presentan seguimiento de por lo menos 2 años, de los cuales 9 (90\%) presentan respuesta sostenida y 1 paciente perdió respuesta y requirió terapia biológica. No se encontró asociación significativa entre la remisión clínica y la extensión de la enfermedad. Ningún paciente requirió colectomía durante el seguimiento.

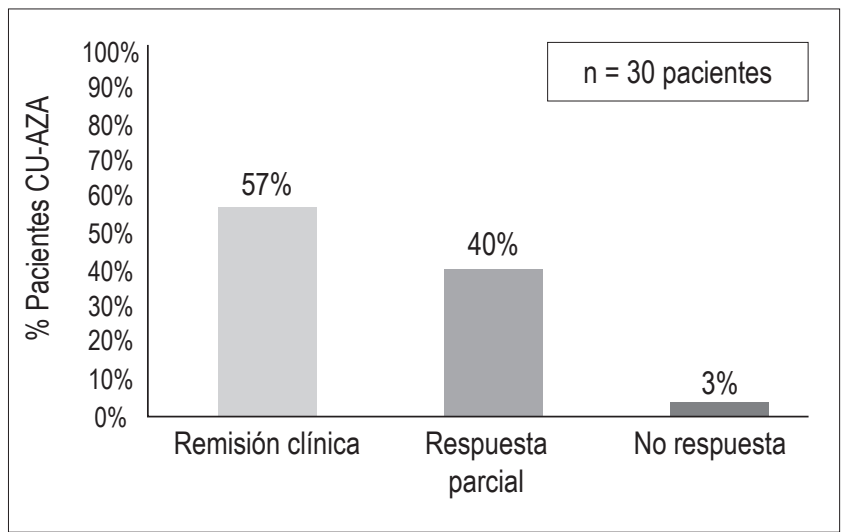

Figura 1. Respuesta clínica a AZA en 3 meses.

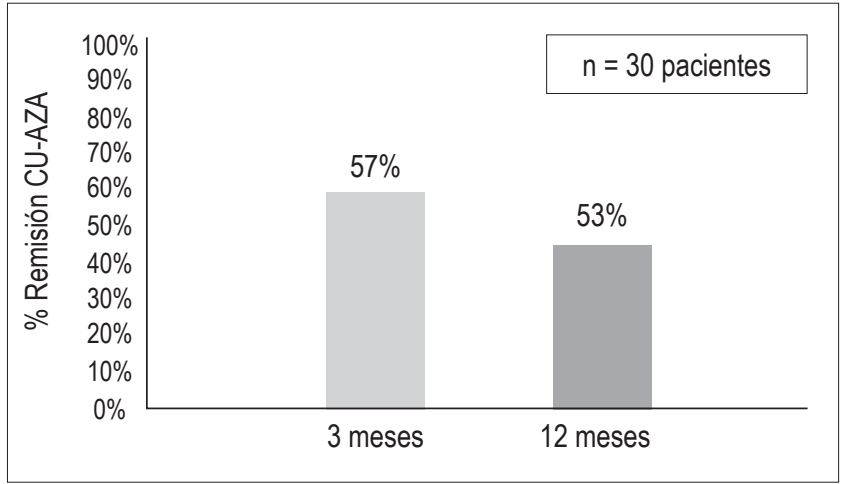

Figura 2. Tasa de remisión CU-AZA.

En cuanto a la seguridad de la azatioprina durante el seguimiento, 6 de los 30 pacientes (20\%) presentaron leucopenia, la cual se resolvió disminuyendo la dosis, sin necesidad de suspender la medicación. Se presentó 1 caso de pancreatitis aguda por lo que se descontinuó AZA en ese paciente, y solo 1 individuo descontinuó la terapia por síntomas gastrointestinales; no se presentó toxicidad hepática, infecciones oportunistas ni linfoma en ninguno de los 30 pacientes durante el seguimiento.

\section{DISCUSIÓN}

Los análogos de purinas como AZA y 6-MP han sido utilizados por más de 30 años para el mantenimiento de la remisión en pacientes con CU refractaria al manejo con 5-ASA y esteroides, o como "ahorradores" de esteroides en pacientes dependientes de estos, con un perfil de riesgo/ beneficio bien establecido, que puede mantener remisión a largo plazo en el $20 \%$ de nuestros pacientes con CU, 
según diferentes investigaciones (15). En nuestro estudio se demostró que AZA es efectiva en el $65 \%$ de los pacientes con CU dependiente o refractaria a esteroides, a 1 año de seguimiento, con un perfil de seguridad adecuado. Este es el primer estudio latinoamericano realizado sobre este tópico en particular.

Los estudios de AZA en el tratamiento de CU son escasos y la mayoría es de hace más de una década. El primero se remonta a 1974, realizado por Jewell y Truelove, donde no logran demostrar que AZA sea de utilidad en inducir ni en mantener la remisión en pacientes con colitis ulcerativa, incluso siendo inferior a la sulfasalazina (16). El primer estudio doble ciego controlado con placebo fue publicado por Hawthorne y colaboradores, en el Reino Unido (17), en sujetos en remisión de CU con AZA por mínimo 6 meses, 33 pacientes con $\mathrm{CU}$ en dosis promedio de AZA de $100 \mathrm{mg} /$ día continuaron la medicación y fueron seguidos a 1 año, encontrando una tasa de recaída de $36 \%$, comparada con $59 \%$ en 34 sujetos a quienes se les suspendió la medicación y recibieron placebo. Otros estudios retrospectivos o abiertos han demostrado tasas de respuesta de $84 \%$ y $63 \%$, y tasas de remisión de $65 \%$ y $69 \%$ (18) en centros de atención terciaria.

La serie retrospectiva más grande se realizó en Oxford con más de 30 años de seguimiento de 346 pacientes, con una tasa de remisión de $58 \%$ (19). Un estudio español más reciente, con 34 pacientes, encontró una tasa de mantenimiento de remisión de 63,6\% en pacientes con 6-MP, y demostraron que el metotrexato era inferior al 6-MP en colitis ulcerativa (20). En un estudio italiano de Ardizzone y colaboradores, se aleatorizaron 72 pacientes con CU dependientes de esteroides a recibir azatioprina $2 \mathrm{mg} / \mathrm{kg} /$ día o 5-ASA 3,2 g/día, encontrando tasas de remisión a 6 meses de 53\% versus $21 \%$, respectivamente, OR: 4,78 (21). Todos estos resultados son similares a los presentados en nuestro estudio, tanto para la respuesta total como para la remisión clínica. Se publicó recientemente una revisión sistemática y metaanálisis de todos los inmunosupresores utilizados en CU; 2 de estos estudios evaluaron AZA en el mantenimiento (130 pacientes), sugiriendo una tendencia al beneficio de la terapia con AZA ( RR $=0,5$, IC 95\% = $0,7-1,0)(22)$. Una revisión sistemática aún más reciente de Cochrane, con 6 estudios que incluyeron 286 pacientes con CU, mostró que AZA es significativamente superior al placebo para mantener la remisión en 51 de 115 pacientes en el grupo de AZA comparado con 76 de 117 pacientes en el grupo placebo (23).

Adicionalmente, existe riesgo de recaída de $\mathrm{CU}$ en individuos a los que se les suspende AZA. Un estudio en pacientes con $\mathrm{CU}$ en remisión clínica que suspendieron AZA, demostró que a 5 años de seguimiento, el $60 \%$ pre- senta recaída de la enfermedad (24). Otro estudio más reciente encontró una tasa de recaída de $26 \%$ a 24 meses en 108 pacientes con $\mathrm{CU}$, a los cuales se les suspendió intencionalmente el fármaco por remisión clínica (25).

Una debilidad del presente estudio es el tamaño de muestra, esto se explica porque muchos de los pacientes que llegan a nuestra Clínica de EII cambian a otro sitio de atención médica y se pierde el seguimiento, por lo tanto no se dispone de información completa; y adicionalmente, algunos de los que llegan han recibido dosis de AZA inferiores de $2 \mathrm{mg} / \mathrm{kg}$, por lo tanto no cumplían los criterios de inclusión y se excluyeron del estudio. Definitivamente, se necesitan más ensayos clínicos, aleatorizados y controlados con placebo, con un mayor número de pacientes, para confirmar los resultados presentados en los diferentes estudios anteriormente señalados.

Los resultados de este estudio son muy importantes porque demuestran que la AZA es un medicamento eficaz en el manejo de individuos con CU con respuesta inadecuada o intolerancia a esteroides, de bajo costo, y con remisión clínica duradera en pacientes que responden y toleran el medicamento, por lo tanto es una alternativa válida previa al manejo con terapia biológica con anti-FNT.

\section{REFERENCIAS}

1. Juliao F, Florez JF, Ruiz MH, et al. Fenotipo e historia natural de la enfermedad inflamatoria intestinal en un centro de referencia en Medellín-Colombia. Rev Col Gastroenterol. 2010;25(3):240-51.

2. Juliao F. Tratamiento médico enfermedad inflamatoria intestinal. Rev Col Gastroenterol. 2007;22(4):313-30.

3. Girardin MM, Manser C, Biederman L, Wanner Roger, Frei Pascal, Safroneeva E, et al. First line therapies in inflammatory bowel disease. Digestion. 2012;86(1):6-10.

4. Dignass A, Lindsay JO, Sturm A, et al. Second European evidence-based consensus on the diagnosis and management of ulcerative colitis Part 2: Current management. J Crohn's and Colitis. 2012;6:991-1030.

5. Faubion WA Jr, Loftus EV Jr, Harmsen WS, et al. The natural history of corticosteroid therapy for inflammatory bowel disease: A population based study. Gastroenterology. 2001;121:255-60.

6. Langholz E, Munkholm P, Davidsen M, Binder V. Colorectal cancer risk and mortality in patients with ulcerative colitis. Gastroenterology. 1992;103:1444-51.

7. Chouchana NC, Beaune P, Loriot MA, Roblin X. The benefits of pharmacogenetics for improving thiopurine therapy in inflammatory bowel disease. Aliment Pharmacol Ther. 2012;35(1):15-30.

8. Ghosh S, Chaudhary R, Carpani M, et al. Is thiopurine therapy in ulcerative colitis as effective as in Crohn's disease? Gut. 2006;55:6-8. 
9. Ginsburg PM, Dassopoulos T. Steroid dependent ulcerative colitis: Azathioprine use is finally "Evidence-Based". Inflamm Bowel Dis. 2006;12(6):921-2.

10. Sands BE. Immunosuppressive drugs in ulcerative colitis: Twisting facts to suit theories? Gut. 2006;55:437-41.

11. Satsangi J, Silverberg MS, Vermeire S, Colombel JF. The Montreal classification of inflammatory bowel disease: Controversies, consensus, and implications. Gut. 2006;55:749-53.

12. Dignass A, Eliakim R, Magro F, et al. Second European evidence-based consensus on the diagnosis and management of ulcerative colitis Part 1: Definitions and diagnosis. J Crohn's and Colitis. 2012;6:965-90.

13. Panaccione R, Rutgeerts P, Sandborn WJ, et al. Review article: Treatment algorithms to maximize remission and minimize corticosteroid dependence in patients with inflammatory bowel disease. Aliment Pharmacol Ther. 2008;28:674-88.

14. Schroeder KW, Tremaine WJ, Ilstrup DM. Coated oral 5-aminosalicylic acid therapy for mildly to moderately active ulcerative colitis: A randomized study. N Engl J Med. 1987;317:1625-9.

15. Louis E, Irving P, Beaugerie L. Use of azathioprine in IBD: Modern aspects of an old drug. Gut. 2014;63(11):1695-9.

16. Jewell DP, Truelove SC. Azathioprine in ulcerative colitis: Final report on a controlled therapeutic trial. BMJ. 1974;4:627-30.

17. Hawthorne AB, Logan RF, Hawkey CJ, et al. Controlled trial of azathioprine withdrawal in ulcerative colitis. BMJ. 1992;305:20-2.

18. Paoluzi OA, Pica R, Marcheggiano A, et al. Azathioprine or methotrexate in the treatment of patients with steroid- dependent or steroid resistant ulcerative colitis: Results of an open-label study on efficacy and tolerability in inducing and maintaining remission. Aliment Pharmacol Ther. 2002;16:1751-9.

19. Fraser AG, Orchard TR, Jewell DP. The efficacy of azathioprine for the treatment of inflammatory bowel disease: A 30 year review. Gut. 2002;50;485-9.

20. Mate-Jimenez J, Hermida C, Cantero-Perona J, et al. 6-Mercaptopurine or methotrexate added to prednisone induces and maintains remission in steroid-dependent inflammatory bowel disease. Eur J Gastroenterol Hepatol. 2000; 12:1227-33.

21. Ardizzone S, Maconi G, Russo A, et al. Randomised controlled trial of azathioprine and 5-aminosalicylic acid for treatment of steroid dependent ulcerative colitis. Gut. 2006;55:47-53.

22. Khurram J, Khan M. Efficacy of immunosuppressive therapy for inflammatory bowel disease: A systematic review and meta-analysis. Am J Gastroenterol. 2011;106(4):630-42.

23. Timmer AMJ, Tsoulis DJ, Macdonald JK. Azathioprine and 6-mercaptopurine for maintenance of remission in ulcerative colitis. Cochrane Database Syst Rev. 2012;9:CD000478.

24. Cassinotti A, Actis GC, Duca P, et al. Maintenance treatment with azathioprine in ulcerative colitis: Outcome and predictive factors after drug withdrawal. Am J Gastroenterol. 2009; 104:2760-7.

25. Kennedy NA, Kalla R, Warner B, et al. Thiopurine withdrawal during sustained clinical remission in inflammatory bowel disease: Relapse and recapture rates, with predictive factors in 237 patients. Aliment Pharmacol Ther. 2014;40:1313-23. 